\title{
Monitoreo de emociones aplicadas a terapias basadas en juegos y lógica difusa para adultos mayores
}

\author{
Monica Janneth Guido Silva, Víctor Manuel Zamudio Rodríguez, \\ Héctor José Puga Soberanes, Juan Martín Carpio Valadez, \\ María del Rosario Baltazar Flores \\ Instituto Tecnológico de León, \\ León, Guanajuato, México \\ m142403@itleon.edu.mx
}

\begin{abstract}
Resumen. La cantidad de personas de edad adulta en México ha aumentado gradualmente, esto ha sido posible gracias al desarrollo médico y tecnológico, lo cual en definitiva es un logro de alto impacto social. Sin embargo con este aspecto, se tiene también un aumento de personas que debido a su edad son más susceptibles a padecer algún tipo de demencia, como el Alzhéimer. Investigaciones recientes están tomando planes de acción y medidas preventivas, ejemplo de ello son las terapias no farmacológicas, como las terapias basadas en juegos del sistema "Mente Activa", el cual es parte de una investigación previa más general y fue desarrollado en el Instituto Tecnológico de León en colaboración con el Instituto de la Memoria, para la detección y prevención del deterioro cognitivo, dicho sistema emplea técnicas de lógica difusa y clasificación. Y genera planes de estimulación cognitiva al evaluar los parámetros como la escolaridad del usuario y los resultados de la prueba neuropsicológica Neuropsi, entre otros. Para mejorar la generación de planes de estimulación, se busca integrar un módulo para el monitoreo de emociones mediante el reconocimiento de expresiones faciales, utilizando el método de base característica, el cual consiste en aplicar técnicas de visión artificial y la extracción de puntos característicos de las regiones faciales de interés. En este trabajo se muestra el reconocimiento de expresiones faciales asociadas a la felicidad, tristeza, sorpresa y un estado normal con personas de edad adulta. Los resultados de la primera etapa del proyecto se muestran alentadores. Posteriormente se añadirá la variable de emoción al sistema de lógica difusa del sistema "Mente Activa".
\end{abstract}

Palabras clave: Alzheimer, cómputo afectivo, demencia, deterioro cognitivo, lógica difusa, reconocimiento de expresiones faciales, terapia asistida por computadora, terapias basadas en juegos.

\section{Introducción}

La población mexicana está experimentando cambios socio-demográficos, como el aumento de la cantidad de personas de mayor edad y el descenso paulatino en el índi- 
ce de mortalidad, de acuerdo con datos del INEGI (Instituto Nacional de Estadística, Geografía e Informática) [1], esto ha sido posible debido a la expansión y mejoras en los servicios de salud, así como al desarrollo en el campo médico y tecnológico, lo cual es un logro social importante, particularmente en México se estima que el porcentaje de adultos mayores incremente a 12.5 por ciento en 2020 y 28.0 por ciento en 2050, para adquirir un perfil propio de las poblaciones envejecidas, de acuerdo con el INAPAM (Instituto Nacional de las Personas Adultas Mayores) [2].

Con este aumento de población se tiene también un incremento en las enfermedades que puede afectar a la misma, como los trastornos cognitivos, la depresión, la ansiedad y las enfermedades crónico-degenerativos [2, 3]. Se estima que a nivel global en 2010 había 35.6 millones de personas con demencia, se prevé que para el 2030 esta cifra se duplicará a 65.7 millones y 115.4 millones en 2050 [3]. Por lo cual la salud mental del adulto mayor emerge como una prioridad social.

Con el propósito de prevenir y contrarrestar dichos problemas de salud mental, han surgido novedosas investigaciones, como las terapias no-farmacológicas, tal es el caso de las terapias basadas en juegos del sistema "Mente Activa", con el cual se puede detectar y prevenir el deterioro cognitivo [4], dicho sistema emplea técnicas de clasificación y de lógica difusa, al evaluar ciertos parámetros (como el grado de escolaridad del usuario y los resultados obtenidos de las pruebas neuropsicológicas; Neuropsi y Minimental), en el módulo inteligente del sistema de "Mente Activa" [5], para generar de manera automática un plan de estimulación individual.

Sin embargo se plantea la cuestión de que los resultados del desempeño puedan verse afectados por el estado emocional del usuario, de acuerdo con el enfoque del Cómputo Afectivo [6]. Por lo que se tiene la propuesta de desarrollar e incorporar un módulo para el monitoreo de emociones, durante la interacción del usuario con los juegos del sistema "Mente Activa". En esta investigación se presenta una breve descripción de los puntos importantes relacionados con el deterioro cognitivo, el cómputo afectivo, un análisis sobre la tecnología usada para mejorar el desempeño cognitivo y el uso de la lógica difusa en el sistema "Mente Activa".

También se presenta el proceso para la implementación del módulo de monitoreo de emociones mediante el reconocimiento de expresiones faciales (como sonrisa, tristeza, sorpresa y un estado normal), aplicando el método de base característica de Gurpreet [7] (el cual aplica técnicas de procesamiento de imágenes y visión artificial, para la extracción de puntos característicos de las diferentes regiones faciales, como ojos y boca).

Posteriormente se muestran los resultados de las pruebas de la primera etapa, correspondiente al desarrollo del módulo para el monitoreo de emociones. Para las pruebas se tomaron imágenes, en tiempo real, de 34 participantes de edad adulta, estas imágenes están asociadas a los estados emocionales de sonrisa, tristeza, sorpresa y un estado normal. Los resultados muestran evidencia de la viabilidad del enfoque. Como trabajo futuro se añadirá la variable "emoción" al sistema de lógica difusa de "Mente Activa", lo cual permitirá mejorar la generación de los planes de estimulación cognitiva individual, fortaleciendo el análisis del desempeño del usuario. 


\section{Deterioro Cognitivo}

Una de las principales discapacidades que se presentan durante la vejez, es la limitación de las habilidades cognitivas.

\subsection{Demencia}

La demencia es una alteración adquirida persistente y progresiva en la función intelectual, que afecta la memoria y el dominio cognitivo generalmente como: afasia (dificultad para elegir palabras), apraxia (incapacidad para llevar a cabo actividades motoras), agnosia (imposibilidad para identificar objetos), alteración de las funciones de ejecución (mala abstracción, flexibilidad mental, planificación y juicio), la depresión y el delirio también es factible que se presenten con daño cognitivo [8].

\subsection{Alzhéimer}

La enfermedad de Alzhéimer es el tipo de demencia más común y generalmente se presenta a partir de los 65 años [9]. Los síntomas más comunes es el deterioro gradual de la habilidad para recordar información reciente. Esto ocurre por el daño en las neuronas, las cuales pueden dejar de funcionar normalmente y mueren, generando cambios en la memoria, el comportamiento y la habilidad de pensar claramente [10].

\section{Cómputo afectivo}

El término "Cómputo Afectivo" fue empleado por primera vez en [11] por Rosalinda Picard, para describir al cómputo que está relacionado con la influencia de las emociones. El estudio del cómputo afectivo, se ha centrado en el reconocimiento, procesamiento e interpretación y simulación de emociones, con la intención de dotar a la máquina con capacidades de comunicación e interpretación afectiva hacía el usuario, ha tomado un gran auge en la implementación de varios sistemas inteligentes, como de tutoría afectiva, aplicación de reconocimiento de emociones mediante expresiones faciales, uso de dispositivos biométricos así como sistemas con fines terapéuticos y psicológicos.

La idea de aplicar cómputo afectivo en el presente proyecto a través del reconocimiento de expresiones faciales, surge de la siguiente premisa: las decisiones tomadas por el ser humano son motivadas por las emociones $[6,11]$, lo cual pudiera afectar el desempeño del usuario durante su interacción con los juegos del sistema "Mente Activa".

\section{1. ¿Qué es una emoción?}

La definición del concepto "Emoción" es una situación no trivial, debido a que existe una gran gama de definiciones en el estado del arte. Kleinginna muestra en su 
artículo un compendio con 92 definiciones [12]. Sin embargo varios investigadores concuerdan en que la emoción es: un conjunto complejo de interacciones entre factores subjetivos y objetivos, mediada por los nervios y la cuestión hormonal [13]. En el presente proyecto se detectará únicamente expresiones faciales relacionadas a los estados emocionales de felicidad, estado normal, sorpresa y tristeza.

\section{Tecnología aplicada a la terapia cognitiva}

En recientes trabajos se han dado a conocer avances tecnológicos para la terapia cognitiva aplicando cómputo afectivo con distintos propósitos como captar el interés de los usuarios, detectar situaciones en que el usuario manifiesta alguna emoción que pueda afectar el rendimiento de su desempeño o influir en algunos aspectos como nivel de atención, aprendizaje, toma de decisiones, etc. Algunos ejemplos de estas investigaciones son: los sistemas de tutoría afectiva [14], la terapia basada en juegos de Neuroracer [15], el reconocimiento de emociones a través de las expresiones faciales [7] y el uso de dispositivos empleados para análisis de las emociones a través de medidas fisiológicas (como la frecuencia cardiaca y la presión arterial) [16].

\section{Sistema inteligente "Mente Activa"}

\section{1. "Mente Activa"}

"Mente Activa" es un programa para la prevención, detección, evaluación y supervisión de los adultos mayores con deterioro cognitivo y la demencia [4]. El sistema permite la estimulación cognitiva a través de la utilización de juegos interactivos especialmente diseñados por psicólogos, el sistema incluye las áreas de estimulación cognitiva como: atención, cálculo, funciones ejecutivas, gnosias, lenguaje, memoria y orientación $[4,5]$. Fue desarrollado en el Instituto Tecnológico de León en colaboración con el Instituto de la memoria, como parte de una investigación más general.

\subsection{Lógica difusa}

La Lógica Difusa es una forma de lógica matemática multivaluada en el que la verdad puede tomar un valor en un rango continuo de valores entre 0 y 1 puesto que utiliza valores lógicos continuos o aproximados entre 0 (completamente falso) y 1 (totalmente cierto). Un enunciado no necesariamente es verdadero o falso, pero puede tener un grado de verdad entre 0 y 1 [19]. Recibe otros dos significados llamados teoría del razonamiento aproximado y la teoría de la lógica lingüística, la última teoría un tanto marginal, es una de las lógicas cuyos valores de verdad son expresiones de lenguaje natural (por ejemplo, cierto, más o menos cierto, etc.) [17]. Zadeh introdujo el término de Lógica Difusa, determinada como un conjunto de principios 
matemáticos para la representación del conocimiento basado en grados de pertenencia [18].
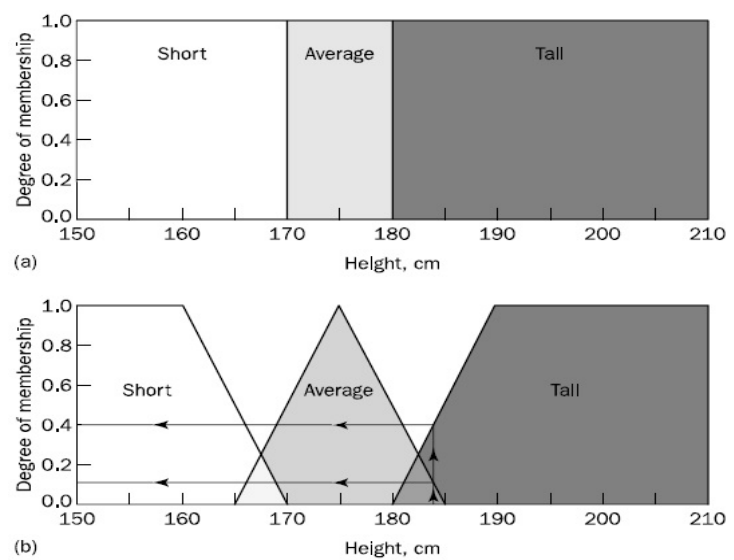

Fig. 1. Esta imagen fue tomada del libro de Inteligencia Artificial de Negnevitsky [19], en este ejemplo de conjuntos difusos se puede apreciar la función de pertenencia donde la variable lingüística altura de un hombre (Height), tiene asociados tres términos lingüísticos (conjuntos difusos): bajo, mediano y alto, para los cuales se tiene asociado un grado de pertenencia, que puede estar entre 0 y 1 . En la imagen (a) se pueden ver los conjuntos de manera cruda, en (b) se muestran los conjuntos difusos.

\subsection{Sistema de lógica difusa tipo 1 (FLS T1) de "Mente Activa"}

Sistema de Lógica Difusa de "Mente Activa" [4] [5], puede ser representado como se muestra en la figura 2. El conocimiento esta incrustado en el conjunto de reglas que alimenta el motor de inferencia del sistema (dadas por los especialistas o psicólogos basándose en antecedentes ó entradas y consecuentes ó salidas difusas). Las entradas en crudo (crisp inputs) consideradas para este sistema son: la escolaridad y la puntuación de los resultados de las pruebas neuro-psicológicas: Neuropsi y Minimental.

Después son fuzzificadas en T1 FSs, que se convierten en nuevos T1 FS por el motor de inferencia. El defuzzificador transforma esta nueva T1 FS en una salida en crudo (crisp output) que es el plan de estimulación cognitiva individual, el cual se pretende mejorar al integrar la nueva variable de entrada "emoción".

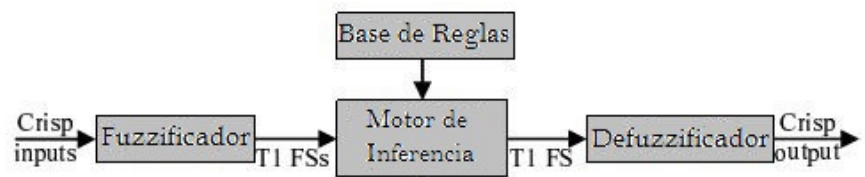

Fig. 2. Esquema de un Sistema de Lógica Difusa tipo 1 de "Mente Activa", tomado de [5]. 


\section{Proceso de implementación del módulo de monitoreo de emociones (mediante el reconocimiento de expresiones faciales)}

La implementación del módulo de monitoreo de emociones está organizada en las siguientes cuatro etapas:

A) Detección del rostro: se utilizó el clasificador "Detect Haar Cascade" de la libreria Emgu CV para C\#. Emgu Cv es un envoltorio para .NET del conjunto de librerías OPENCV [20], con la cual se puede hacer procesamiento de imágenes y aplicar técnicas de visión artificial. Su aplicación puede verse en Figura 3.

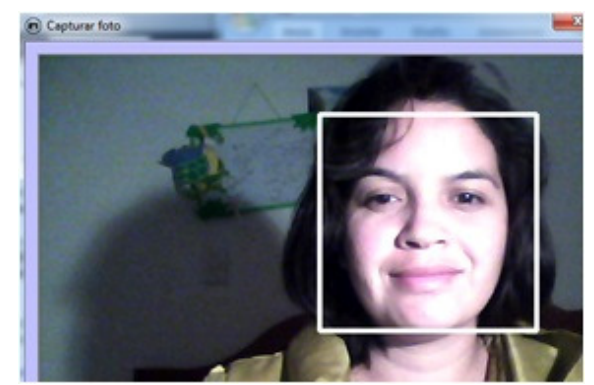

Fig. 3. Ejemplo de la detección del rostro aplicando la librería Emgu CV, la cual se encuentra disponible en [20].

B) Reconocimiento de Emociones a través de las expresiones faciales: se aplicó el método de base característica, para detectar expresiones faciales humanas, el cual puede ser estudiado con mayor detalle en $[7,21]$. De manera general el procedimiento que emplea dicho método es el siguiente:

- Pre-procesamiento de la imagen obtenida en la detección del rostro: Se obtiene una imagen RGB (del inglés de red, green, blue), la cual es contrastada y transformada en una imagen en blanco y negro, después se realiza la obtención del rostro como área del interés.

- Se buscan el centro de la imagen y se aplica el patrón de seguimiento al desplazarse por cada punto, para detectar los ojos y la boca como nuevas áreas de interés en la imagen.

- Posteriormente se aplican las curvas de Bezier en las áreas de boca y ojos extrayendo los puntos característicos faciales.

- Finalmente la representación de los valores de los puntos obtenidos son comparados en la base de datos que contiene previamente los vectores de puntos asociados con las emociones de sonrisa, tristeza, sorpresa y un estado normal. Por lo que esta clasificación no requiere de un entrenamiento previo.

La técnica de base característica se lleva a cabo detectando cambios característicos en las diferentes regiones faciales, dichas regiones están basadas en el 
Sistema de Codificación de la Acción Facial (FACS: Facial Action Coding System). El cual es un sistema basado en la observación humana y permite detectar cambios en las características faciales [7].

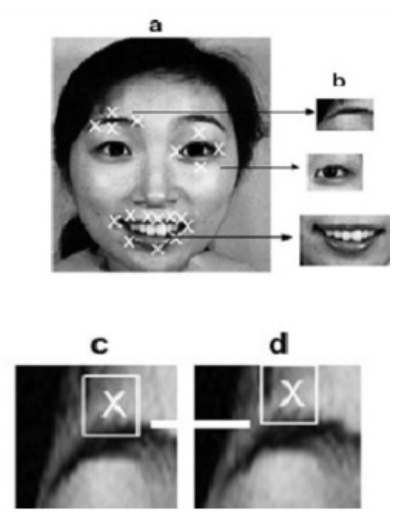

Fig. 4. Imagen, tomada de [21], muestra el proceso para el reconocimiento de emociones a través de las expresiones faciales, aplicando el método de base característica de Gurpreet [7].

Como puede verse en la Fig. 4, "a" Una vez detectado el rostro se procesa la imagen, "b" se extraen las características faciales, "c" y "d" el patrón de seguimiento permite encontrar las regiones de interés. Para el caso particular de esta investigación solo se extrajeron las regiones de interés correspondientes a los ojos derecho e izquierdo y la boca.

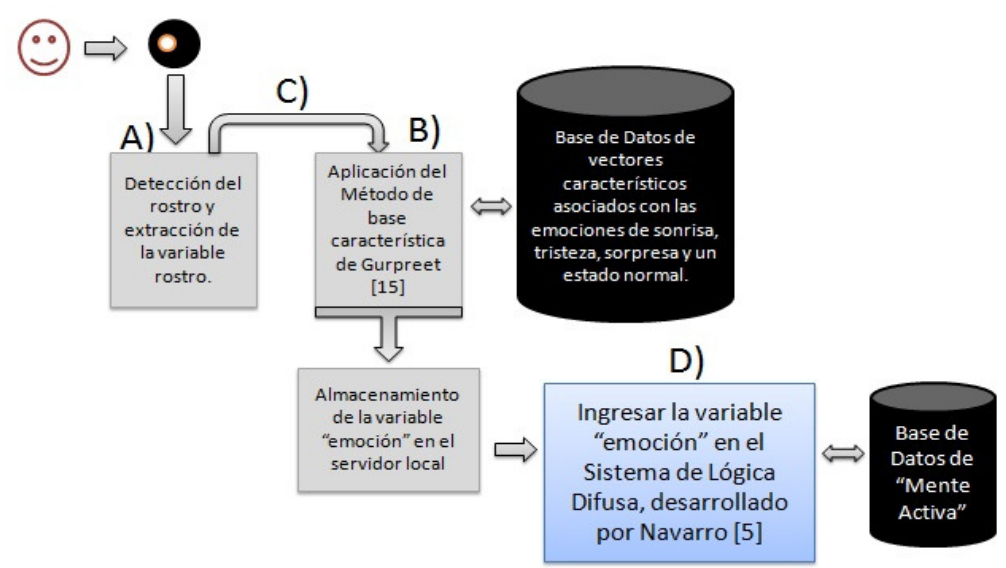

Fig. 5. El estatus actual de la investigación se encuentra en la etapa de integración de las etapas: a) detección de rostro, b) reconocimiento de emociones a través de expresiones faciales. 
C) Integración de las primeras dos etapas: Incisos A y B, en la Figura 5, se muestra el diagrama de integración.

D) Ingresar la variable de la emoción detectada en el FLS T1 [4] [5] de "Mente Activa". Finalmente la variable "emoción" obtenida será añadida al sistema de lógica difusa de "Mente Activa" como se muestra en el inciso D) de la figura 4, esto permitirá mejorar la generación de planes de estimulación cognitiva individual.

\section{Experimentación}

Para realizar las pruebas al módulo de monitoreo de emociones mediante el reconocimiento de expresiones faciales, se tomaron 34 imágenes para cada categoría (felicidad, tristeza, sorpresa y normal) a una muestra de 34 participantes de edad adulta, este tamaño de muestra para el experimento es de acuerdo al teorema del límite central $[23,24]$, para un total de 136 muestras para clasificación. Las imágenes fueron tomadas en tiempo real con la cámara digital de computadora. Para el cálculo de la precisión se empleó la siguiente ecuación, propuesta en [22]:

$$
\text { Precision }=\frac{+P}{(+P)+F P}
$$

donde $+\mathrm{P}$ son los verdaderos positivos y FP son los falsos positivos.

A continuación se muestra el porcentaje de precisión del monitoreo de emociones a través de expresiones con imágenes tomadas con la cámara.

Tabla 1. Porcentaje de precisión del reconocimiento de emociones a través de las expresiones faciales con imágenes tomadas de la cámara digital de la computadora con personas de edad adulta. Los números 27, 28, 20 y 28 son los verdaderos positivos de acuerdo con la fórmula de precisión [22].

\begin{tabular}{lccccc}
\hline Categorías & Normal & Feliz & Triste & Sorpresa & Precisión \\
\hline Normal & 27 & 2 & 5 & 0 & $79.41 \%$ \\
Feliz & 2 & 28 & 0 & 4 & $82.35 \%$ \\
Triste & 10 & 0 & 20 & 4 & $58.82 \%$ \\
Sorpresa & 2 & 4 & 0 & 28 & $82.35 \%$ \\
\hline
\end{tabular}

\section{Conclusiones}

En este trabajo de investigación se muestra el diseño y desarrollo del módulo para el monitoreo de emociones basado en el estudio del Cómputo Afectivo mediante el 
reconocimiento de expresiones faciales, el cual será integrado a un sistema de terapias basadas en juegos y lógica difusa para Adultos Mayores llamado "Mente Activa".

Para las pruebas realizadas en la primera etapa del módulo se tuvo una muestra de 34 usuarios de edad adulta, tomando un total de cuatro imágenes por persona, cada una correspondiente a las categorías de expresiones faciales asociadas a la felicidad, tristeza, sorpresa y un estado normal, posteriormente dichas imágenes fueron procesadas con el "método de base característica para detectar expresiones faciales humanas" que emplea técnicas de procesamiento de imágenes y de visión artificial.

Los resultados de la experimentación muestran la viabilidad del enfoque, sin embargo las condiciones de experimento son cruciales (como la iluminación, calidad de la imagen, la disposición de participación del usuario, los movimientos bruscos del usuario, entre otras), ya que afectan el índice de clasificación.

El trabajo futuro propuesto es realizar más pruebas con usuarios de edad avanzada y finalmente dicha representación de la variable del estado emocional (variable "emoción") se añadirá en una segunda etapa donde pueda ser utilizada como una variable de entrada, en el sistema de lógica difusa de "Mente Activa", y así poder mejorar la generación de planes de estimulación cognitiva y fortalecer el análisis del desempeño del usuario.

Agradecimientos. Agradecimientos Al Consejo Nacional de Ciencia y Tecnología (CONACYT) de México, por el apoyo brindado en esta investigación y a la División de Estudios de Posgrado e Investigación del Instituto Tecnológico de León.

\section{Referencias}

1. Instituto Nacional de Estadística, Geografía e Informática: Los Adultos Mayores en México; Perfil socio demográfico al inicio del siglo XXI. Aguascalientes, México: INEGI. Disponible en www.inegi.gob.mx, pp. 9-18 (2005)

2. INAPAM: Instituto Nacional de las Personas Adultas Mayores; Gobierno Federal, Ejes reactores de la política pública nacional a favor de las personas adultas mayores, pp. 13$35,49(2010)$

3. Gutiérrez Robledo, L. M.: Plan de Acción Alzheimer y Otras Demencias. México 2014, Instituto Nacional de Geriatría, pp. 19-54 (2014)

4. Navarro, F. J., Zamudio, V., Baltazar, R., Lino, C., Díaz, C., Faiyaz D.: Towards Game Based Monitoring and Cognitive Therapy for Elderly using a Neural-Fuzzy approach. In: Workshops Proceedings in the Mexican International Conference on Computer Science, pp. 1-6 (2013)

5. Navarro, F. J.: Sistema Inteligente para el Monitoreo y Generación de Tareas en Escenarios de Terapia para Adultos Mayores. Instituto Tecnológico de León, León Guanajuato, México, pp. 51-63 (2014)

6. Picard, R. W.: Online Affective computing: challenges. International Journal of Human Computer Studies, vol. 59, pp. 55-64, Elsevier Science Ltd., Cambridge, DOI: 10.1016/S1071-5819(03)00052-1 (2003)

7. Gurpreet, S., Vikas, W.: Feature Based Method to detect Human Facial Expressions. International Journal of Computer Science and Communication Engineering, IJCSCE, India, pp. 42-47 (2013) 
8. McPhee, S. J., Papadakis, M.A.: Diagnóstico clínico y tratamiento. McGraw-Hill, México, pp. 63-65 (2012)

9. Sosa, A. L., Acosta, I.: Epidemiología de las Demencias. Instituto Nacional de Geriatría, México, Sitio web http://www.geriatria.salud.gob.mx (2014)

10. Alzheimer's Association: Alzheimer's Disease Facts and Figures. Alzheimer's and Dementia, Volume 10, Issue 2, pp. 5-14 (2014)

11. Picard, R. W.: Affective Computing. MIT, Media Laboratory Perceptual Computing Section Technical Report No. 321, pp. 1-16 (1998)

12. Kleinginna, Jr. P. R., Kleinginna, A. M.: A Categorized List of Emotion Definitions, with Suggestions for a Consensual Definition. Motivation and Emotion, vol. 5, no. 4, pp. 345379 DOI: 0146-7239/81/1200-0345 (1981)

13. Van den Broek, E. L.,Janssen, J. H.,\& Westerink, J. H.: Guidelines for Affective Signal Processing (ASP): From Lab to Life. In: 3rd International Conference on Affective Computing \& Intelligent Interaction, Amsterdam, The Netherlands, pp. 704-709 (2009)

14. Mao, X., Li, Z.: Agent based affective tutoring systems: A pilot study. Computers and Education, 55, pp. 202-208 (2010)

15. Anguera, J. A., Boccanfuso, J., Rintoul, J. L., Al-Hashimi, O., Faraji, F., Janowich, J. et al.: Video gaming enhances cognitive skills that decline with age: Game Change. Macmillan Publishers, San Francisco, California, pp. 97-10 (2013)

16. Aguiñaga, A. R., López, M., Alanís, A., Baltazar, R. \& Zamudio, V. M.: Emotion analysis through physiological measurements. In: J. Botía, \& D. Charitos (Ed.), Workshop Proceedings of the $9^{\text {th }}$ International Conference on Intelligent Environments, 17, pp. 97106, IOS Press (2013)

17. Novák, V., Perfilieva, I. and Močkoř, J.: Mathematical principles of fuzzy logic Dordrecht. Kluwer Academic (1999)

18. Zadeh, L.: Fuzzy sets. Information and control, vol. 8, no. 3, pp. 338-353 (1965)

19. Negnevitsky, M.: Artificial Intelligence. A Guide to Intelligent Systems. Pearson Education, pp. 88-93 (2002)

20. EmguCv, Disponible en http://www.emgu.com/wiki/index.php/Main_Page (2015)

21. S. Gurpreet, Baljit, S.: Feature Based Method for Human Facial Emotion Detection using Optical Flow Based Analysis. An International Journal of Engineering Sciences, Journal Anu Books, vol. 4 (2011)

22. Fawcett, T.: An Introduction to ROC analysis. Pattern Recognition Letters, Elsevier, vol. 27, pp. 861-874 (2006)

23. Grinstead, Ch. M.; Snell, J. Laurie: Introduction to Probability. AMS Bookstore, pp. 325360 (1997)

24. Filmus, Y.: Two Proofs of the Central Limit Theorem. pp. 1-3 (2010) 